\title{
A space mission to detect imminent Earth impactors
}

\author{
G. B. Valsecchi ${ }^{1,3}$, E. Perozzi ${ }^{2,1}$ and A. Rossi ${ }^{3}$ \\ ${ }^{1}$ IAPS-INAF, Roma, Italy \\ ${ }^{2}$ Deimos Space, Madrid, Spain \\ ${ }^{3}$ IFAC-CNR, Sesto Fiorentino, Italy
}

One of the goals of NEO surveys is to discover Earth impactors before they hit. How much warning time is desirable depends on the size of the impactors: for the larger ones more time is needed to mount effective mitigation measures. Initially, NEO surveys were aimed at large impactors, that can have significant global effects; however, their typical time scale is orders of magnitude larger than human lifetime. At the other extreme, monthly and annual events, liberating energies of the order of 1 to 10 kilotons, are immaterial as a threat to mankind, not justifying substantial expenditure on them. Intermediate events are of more concern: in the megatons range, timescales are of the order of centuries, and the damage can be substantial. A classical example is the Tunguska event, in which a body with a diameter of about 30 to $50 \mathrm{~m}$ liberated about 5 megatons in the atmosphere, devastating 2000 square kilometers of Siberian forest.

Tunguska-class impactors are faint, not easy to discover and track for ground-based surveys; if such an impactor were to approach the Earth from the direction of the Sun, it could not be discovered telescopically, as it would be in the daytime sky. However, if it would make pre-impact approaches to the Earth, it could be discovered during one of them, allowing for tracking and orbit determination. A NEO survey then would, after an initial period during which it would be "blind" with respect to immediate impactors coming from the direction of the Sun, have discovered and tracked a sufficiently large number of Tunguska-class NEOs that the next impactor would already be among the discovered ones at the time of fall. Farnocchia et al. (2012) simulated such a process, concluding that the survey envisaged for the NEO Segment of the SSA Program of ESA would almost reach a $90 \%$ probability of discovering the next Tunguska-class impactor before impact in about one century from start. Thus, there is room for an alternative survey strategy for discovering Tunguska-sized impactors during the period of time in which ground-based surveys complete the catalog of impactors coming from the direction of the Sun. This strategy has to spot impactors while they are just inside the Earth orbit.

Let us consider a NEA with a geocentric speed of $15 \mathrm{~km} / \mathrm{s}$; if it approaches the Earth from the direction of the Sun, it would spend about 11.5 days within the last $0.1 \mathrm{AU}$ of its trajectory towards the Earth. If discovered from space, when still at $0.1 \mathrm{AU}$ from our planet, this would give enough time for an effective evacuation of the impact area.

The crucial question is: in what orbit do we put the spacecraft? Earth-bound orbits allow to observe close to the Sun, but their advantage over ground telescopes in terms of warning time for an imminent impactor is negligible. Satellites at $\mathrm{L}_{1}$ would be more efficient than those in Earth orbit, but the warning time would still be too short. If the spacecraft is put in a heliocentric orbit interior to that of the Earth, its sensor can point in the anti-solar direction, to spot objects when their brightness is near maximum due to the small phase angle. However, the drawback would be that the difference of heliocentric 


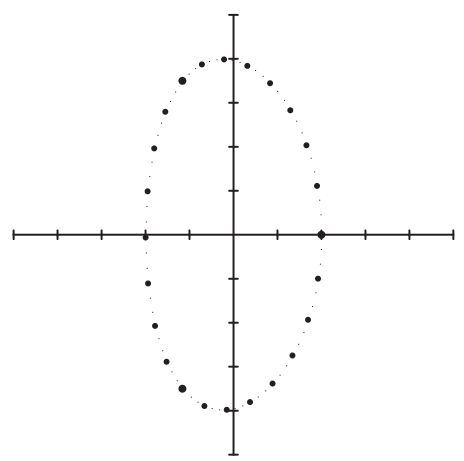

Figure 1. The motion of the 3 spacecraft in a geocentric rotating frame (Sun on the negative $x$-axis); the tic marks are spaced by $0.05 \mathrm{AU}$, and the 3 largest dots (at $x=0.1, y=0$, at $x=-0.06, y=-0.17$, at $x=-0.06, y=0.17$ ) show the 3 spacecraft at a certain epoch. After that, their motion is clockwise; small dots show the positions every 5 days, and intermediate size dots show those every 15 days.

longitude between the spacecraft and the Earth would go from $0^{\circ}$ to $180^{\circ}$, making the communications difficult and impairing to ability to spot imminent impactors.

We propose to put the spacecraft in an orbit like those studied by Hénon $(1969,1970)$, resembling a distant retrograde Earth satellite. These are heliocentric orbits in which the semimajor axis is almost exactly $1 \mathrm{AU}$ and the inclination is small; the eccentricity can be chosen within a rather large range, and for our purposes we can choose a value of 0.1 .

A spacecraft moving on such an orbit would:

(a) have the same orbital semimajor axis (1 AU), and hence the same orbital period (1 yr) as the Earth;

(b) undergo a radial excursion from the Sun equal to twice its eccentricity (for $e=0.1$, it would have $q=0.9 \mathrm{AU}, Q=1.1 \mathrm{AU})$;

(c) undergo an excursion in longitude such that it would, at the extrema, precede or follow the Earth by about twice the value of its orbital eccentricity, expressed in AU.

Seen from Earth, the spacecraft would make a retrograde orbit about it, with minimum geocentric distance, at inferior and at superior conjunction, equal to the value of its orbital eccentricity expressed in $\mathrm{AU}$, and maximum geocentric distance, at quadratures, equal to twice the value of its orbital eccentricity, again in AU. In such an orbit, even a relatively small telescope would be very efficient in monitoring the region immediately interior to the Earth orbit, when moving at heliocentric distance less than 1 AU, but would be inefficient otherwise. To address this problem, a possible solution is the insertion of three spacecraft, appropriately spaced, in the same orbit. In this way, there would be at any time at least 1 spacecraft optimally placed for impactor monitoring. In the following, we assume to have 3 spacecraft moving on an orbit with semimajor axis equal to $1 \mathrm{AU}$, $e=0.1$, and very small $i$, of the order of $0.1^{\circ}$.

A small constellation of 3 satellites properly spaced along the same "distant retrograde satellite" orbit could be quite effective in monitoring imminent small impactors, ensuring a reasonable warning time. These satellites could of course be very effective also in helping to complete the catalogue of low-MOID small NEOs.

\section{References}

Farnocchia, D., Bernardi, F., \& Valsecchi, G. B. 2012, Icarus 219, 41

Hénon, M. 1969, A\&A 1, 223

Hénon, M. 1970, A\&A 9, 24 